Canadian Science Publishing

Journal of Unmanned Vehicle Systems Revue des systèmes de véhicules télécommandés

\title{
Development of a low-cost multispectral camera for aerial crop monitoring
}

\begin{tabular}{|r|l|}
\hline Journal: & Journal of Unmanned Vehicle Systems \\
\hline Manuscript ID & juvs-2017-0008.R1 \\
\hline Manuscript Type: & Note \\
\hline Date Submitted by the Author: & 03-Jul-2017 \\
\hline Complete List of Authors: & $\begin{array}{l}\text { Barrows, Clayton; Aerial Precision Images } \\
\text { Kulanon, Duke; Northwest Nazarene University, }\end{array}$ \\
\hline Keyword: & Crop monitoring, multispectral imaging, unmanned aerial vehicle \\
\hline $\begin{array}{r}\text { Is the invited manuscript for } \\
\text { consideration in a Special } \\
\text { Issue? : }\end{array}$ & N/A \\
\hline
\end{tabular}

\section{SCHOLARONE ${ }^{\text {TH }}$}

Manuscripts 


\title{
Development of a low-cost multispectral camera for aerial crop monitoring
}

\author{
Clayton Barrows \\ Aerial Precision Images, LLC \\ Star, ID \\ Duke M Bulanon \\ Department of Engineering \\ Northwest Nazarene University \\ Nampa, ID
}

\begin{abstract}
This paper presents the development of a low-cost multispectral camera that can be mounted on small unmanned aerial vehicles for crop monitoring. The low-cost multispectral camera is a modified commercial grade point-and-shoot camera with a special dual-band filter that can detect narrow red band and near-infrared band. The modified camera was evaluated by flying it over a commercial apple orchard, and its performance was compared with a commercial multispectral camera. Results showed that the modified camera produced a similar Normalized Difference Vegetation Index images as compared with the commercial multispectral camera.
\end{abstract}

Keywords: Crop monitoring, multispectral imaging, unmanned aerial vehicle 


\section{Introduction}

It is estimated that to feed the world population in 2050, our current crop production will need to double (Food and Agriculture Organization 2009). One step towards realizing this is by applying precision agriculture techniques that have the ability to maximize yields. Precision Agriculture (PA) is a site-specific technology with the goal of maximizing output while minimizing input (Lee et al. 2010). Examples of these precision agriculture applications are soil sensing, fertilizer application, and yield monitoring. Monitoring crop health frequently during the growing season and detecting early signs of stress related to water and nutrient deficiencies as well as pest infestations is critical in optimizing the productivity of a crop (Alchanatis et al. 2010).

Although precision agriculture has been commercially available for row crops for quite a while now, the application of precision agriculture for specialty crops is still in the research phase. Specialty crops include fruits, vegetables, nuts, and ornamental plants (Burks et al. 2008). The challenges of specialty crops like fruit trees are the physical size and configuration of the plants. To cite an example, in monitoring row crops using a camera, the camera can easily be mounted on a tractor and images of the field can easily be collected as the tractor moves across the field (Kataoka et al. 2004). It is difficult to adopt this technique for fruit trees.

However, with the availability of small unmanned aerial vehicles (UAV), collecting images from fruit trees can be realized. Unmanned Aerial Vehicles are accessible, economical, and timely method of carrying sky based sensors to measure the reflected light from plants (Cano et al. 2017). Before the advent of UAV, remote sensing for crops utilized either manned aircrafts or satellites. The disadvantages of both systems are higher cost, lower resolution, and low frequency of imaging. The use of UAV combined with miniaturized imaging system alleviates the disadvantages of the expensive remote sensing alternatives.

The challenges for farmers in using small UAV-based imaging are 1) the processing and analysis of tons of images, 2) the type and cost of the imaging sensor, and 3) the cost and technical operation of small UAV. However, some commercial companies have released easy to use software applications combined with off-the-shelf UAV that facilitates the use of UAV-based imaging. One of these companies is DroneDeploy (DroneDeploy 2017). DroneDeploy developed apps for iOS and Android platforms that allow users to plan the flights, fly the small UAV autonomously, collect images, and process the images. Many of the cameras that come with an off-the-shelf small UAV are RGB cameras, which do not use narrow band filters or offer the ability to look at specific wavelengths (Boon et al. 2016). One of the current monitoring methods involves using costly specialized image sensors that can sense specific wavelengths of red and near-infrared that are reflected from crops (Yang et al. 2004). Garcia-Ruiz et al. (2013) used a six narrow-band multispectral camera to monitor citrus orchards. This is effective because vegetation will reflect these wavelengths in a predictable manner in relation to stress. Healthy plants normally absorb a good amount of red light and reflect a lot of NIR light. When crops are stressed they will begin to reflect more red and absorb more NIR than a healthy crop. Monitoring these wavelengths also provides the possibility to see changes in crop health before they can be seen visually. Sankaran et al. (2011) investigated the visible-near infrared reflectance of citrus to detect the presence of citrus greening. These multispectral sensors measure the amount of red and near-infrared light reflected from the crop and then the reflectance values are used to calculate the Normalized Difference Vegetation Index (NDVI) (Koller and Upadhyaya 2005), 
which can be correlated to crop health.

$$
N D V I=\frac{N I R-R e d}{N I R+R e d} \quad(\text { Eq. } 1)
$$

Although these sensors are effective in monitoring crop health, it is currently cost prohibitive for farmers and invalidates the cost-saving of crop monitoring (McCabe et al. 2016). This paper presents the development of a low-cost multispectral camera that can be mounted on off-theshelf UAV. The low-cost multispectral camera is a single-sensor modified commercial grade camera that has the ability to detect red and near-infrared. The objectives of this study are 1) to develop a low-cost multispectral camera and 2) to compare the performance of this camera with a commercial multispectral camera, specifically the Micasense RedEdge 3.

\section{Methodology}

\section{Multispectral Sensors}

\section{Low-Cost Multispectral Camera}

The low-cost multispectral camera (LCMC) is based on the Canon S100, which is a compact 12megapixel consumer camera (Figure 1). It has RAW and JPEG support and controllable settings of the ISO, shutter speed, and aperture. It has an on-board GPS sensor. It employs a CMOS sensor, which is typically sensitive to wavelengths between $350 \mathrm{~nm}-1050 \mathrm{~nm}$. A dual-band filter from Midwest Optical Systems (MidOpt 2017) is used to allow Red wavelength centered around $660 \mathrm{~nm}$ and NIR wavelength centered around $850 \mathrm{~nm}$ to pass to the sensor. This dual-band filter has narrower bands as compared with other common filters options for near-infrared imaging of crops (Event38 2017; Llewellyn Data Processing 2017).

Figure 1 Canon S100 Camera with calibration targets

The Canon S100 was modified by removing the internal factory infrared filter and replacing it with the MidOpt DB660/850 narrow dual bandpass filter (Midwest Optical Systems 2017). This will provide the red band and the NIR band, which are the bands needed to calculate NDVI. There will be some near-infrared contamination in the red channel, which is typical of a single sensor NDVI imager. This is due to the fact that all recorded channels of the Canon camera are sensitive to NIR light once the factory IR filter is removed.

The Canon S100 had a ground sampling distance of $3.21 \mathrm{~cm} / 1.26 \mathrm{in}$ from a distance of 300 feet above the ground. All images were calibrated and optimized in Pix4D Pro. There was a 2.8\% relative difference between initial and optimized internal camera parameters. Images were clear with uniform lighting throughout the data set. The Canon camera captures two bands, red and NIR, used to compare in this study.

\section{Micasense Camera}

The Micasense RedEdge 3 is a 12-bit RAW multispectral imager with five sensors at 1.6 
megapixel resolution (MicaSense 2017). The only bands used in this study were the red at 668 $\mathrm{nm}$ and NIR at $840 \mathrm{~nm}$. Each sensor has narrow bandpass filter and RAW image format support. It has automatic or user configurable control over exposure and gain. It has an attachable GPS sensor, magnetometer, and irradiance sensor to record angle during image capture as well as irradiance from the sun overhead. Micasense recommends leaving camera settings on auto mode.

The Micasense RedEdge 3 had a ground sampling distance of $6.18 \mathrm{~cm} / 2.43$ in at 300 feet above the ground. All images were calibrated and optimized in Pix4D Pro (Pix4D 2017). There was a $1.11 \%$ relative difference between initial and optimized internal camera parameters. Images were clear with uniform lighting throughout the data set. The Micasense camera captures five bands in each image set. Only the red and NIR bands were used in this study to match the bands used with the Canon S100.

Figure 2 Micasense RedEdge Multispectral Camera

\section{Camera Settings, Image Acquisition, and Processing}

The Micasense exposure and gains settings were left on auto based on manufacturer recommendation. The Canon Camera settings were set at ISO 100, Aperture f2.8, and shutter speed 1/2000 for all images. Both sets of camera images were calibrated against standard reflectance panels with known reflectance values. The reflectance values used were for the red and NIR wavelengths for each camera respectively. Only RAW images were used. The images were obtained on the same day at around solar noon. Images of the calibration target were acquired immediately prior to each flight with each camera. The flight with the Canon camera was flown within five minutes of landing with the Micasense camera. The image sets were processed with Pix4D Pro using the calibration option. This consisted of drawing a region of interest on the image of the calibration target and applying the known values for given wavelengths. The NDVI equation (Eq 1)was then applied to each set of calibrated images with the only difference being a modification to the NDVI equation with the Canon images. This was done to account for the NIR contamination in the red channel (Fredembach and Susstrunk 2008). The modified formula consisted of subtracting a percentage of the NIR pixel values from the Red pixel values. This percentage was an empirical value based on field measurement of specific areas within the orchard taken with the handheld Trimble Greenseeker and comparison to the values produced by the Micasense imaging. The formula was adjusted until the closest match of NDVI values was accomplished. Equation 1 is used to calculate NDVI for the Micasense camera.

The modified NDVI equation for the Canon camera is

$$
\text { modified NIR }=\frac{N I R-(\text { Red }-N I R(0.4))}{N I R+(\text { Red }-N I R(0.4))}(\text { Eq. 2) }
$$

The sun produces varying amounts of radiance through different wavelengths, and the camera sensor also has varying sensitivities to different wavelengths. To correct for this, images of radiometric calibration targets were taken immediately before each flight with each respective sensor. Use of this target allows for correction of variances in wavelength intensity during image acquisition. In order to obtain images with full reflectance values from the sun at nadir, the UAV 
was flown and images acquired within one hour of solar noon for the test orchard location. The flights were performed on days with clear skies free of clouds.

Cameras were attached pointing straight down (nadir) in the UAV using a camera hatch assembly. This consists of a servo powered cargo door that stays closed during takeoff and landing, and opens during image capture. The cameras were set to take images approximately every second.

\section{Target Field}

The images used in this study were acquired from Tylers Rocky Point Orchard in Emmett, Idaho. The fruit orchard is composed of Fuji, Red Delicious, and Pink Lady apple varieties. They are grown in a sandy loam soil with irrigation from a hand set line that moves through every 3-4 weeks.

\section{Small Unmanned Aerial Vehicle}

The UAV used to acquire images for this study was the Event38 E-384 fixed wing UAV(figure 3) with programmable autopilot functions and capable of flying grids at controlled altitude and coordinates to image specific areas. Mission planning is accomplished using the Tower app on a handheld tablet. The region to be imaged is circled on the current map displayed on the tablet. The app then automates a survey grid according to the desired altitude and overlap. Automatic take-off and landing is programmable in the mission, leaving the operator free to monitor the UAV's operation and make flight adjustments if necessary.

Figure 3 E384 UAV

\section{Results and Discussion}

Figure 4(a) shows the apple orchard that was used in this study and a ground image of one of the apple trees. The zoomed images, figures 4(b) and 4(c), of the outlined area are the Canon false color image and the MicaSense color image, respectively. The Canon false color image is a combination of the red band (red channel), NIR band (green channel), and NIR band (blue channel), which produced the false color where the trees show a blue color. On the other hand, the MicaSense color image is a combination of the red band (red channel), green band (green channel) and blue band (blue channel) producing a true color image.

Figure 4 Target Apple Orchard

To calculate the NDVI, only the red band and the NIR band are used and these are the two bands 
that are focused in this study. The gray scale images of the red and NIR bands from the two cameras are shown in figure 5. It can be observed in the red band images of the two cameras that the tree canopies have darker intensity than the ground. This is because vegetation absorbs the red band and reflect the NIR band. The reflection of the NIR band is shown in the NIR images where the tree canopies appear brighter than the ground in both cameras. However, there is a difference of contrast between the two cameras in both the red band and NIR images. The MicaSense has a higher contrast than the Canon in the red band images, while the Canon has a higher contrast in the NIR band image. This contrast difference is a result of the different sensitivity between sensors and the optical band filters that were used. The Canon has a single sensor with a dual band filter while the MicaSense has five sensors with five different bandpass filters. The single sensor of the Canon also contributed to the NIR contamination with using a dual band filter, which is the reason for the modification of the standard NDVI equation.

Figure 5 Gray Scale Images of red band and near-infrared band

Figure 6 shows the NDVI images of both cameras. It can be observed that the NDVI image of the Canon is comparable to the NDVI image of the MicaSense. The tree canopies from the Canon camera have an average NDVI of $0.77 \pm 0.11$ while the NDVI of the tree canopies from MicaSense averaged $0.79 \pm 0.09$. Although the red image and NIR image of both cameras had different intensity values, this was compensated by using the same radiometric targets for both cameras and modifying the NDVI equation for the Canon camera. A t-test conducted to compare the NDVI of the Canon and MicaSense showed that there was no significant difference in the values $(\mathrm{t}(14)=2.14, \mathrm{p}=0.74)$.

The results of this comparative study will be very useful for the farmers because it will allow them to use a low-cost sensor that provides similar NDVI images acquired from a true multispectral sensor. It has the potential to conduct crop monitoring at a much lower cost by using small UAVs combined with a modified point-and-shoot camera.

Figure 6 NDVI Images of both cameras

\section{Conclusion}

This study presented the development of a low-cost multispectral camera that can be used to estimate NDVI for crop monitoring using UAVs. The low-cost multispectral camera is a modified commercial grade single-sensor camera with a special dual-band filter that could detect the red band and near-infrared band. The modified camera was attached to a fixed wing UAV and flown over a commercial apple orchard. The performance of the camera, with respect to NDVI estimation, was compared with a commercial multispectral camera, MicaSense RedEdge 3 , that was flown on the same orchard. The modified camera produced a comparable NDVI false color image with no significant difference as the commercial multispectral camera, which will be useful for orchard monitoring. The results of this comparative study provide a cost-effective multispectral imaging using an unmanned aerial vehicle system. 
Acknowledgement

This research was supported by the Idaho State Department of Agriculture (Idaho Specialty Crop Block Grant), Northwest Nazarene University, and Tylers Rocky Point Orchard.

References

Alchanatis, V., Cohen, Y., Cohen, S., Moller, M., Sprinstin, M., Meron, M., Tsipris, J., Saranga, Y., and Sela, E. 2010. Evaluation of different approaches for estimating and mapping crop water status in cotton with thermal imaging. Precision agriculture, 11(1), pp. 27-4.

Boon, W.A., Greenfield, R., and Tesfamichael, S. 2016. Wetland Assessment Using Unmanned Aerial Vehicle (UAV) Photogrammetry. The International Archives of the Photogrammetry, Remote Sensing and Spatial Information Sciences, Volume XLI-B1, 2016, XXIII ISPRS Congress, 12-19 July 2016, Prague, Czech Republic.

Burks, T.F., Schmoldt, D.I., and Steiner, J.J. 2008. U.S. Specialty Crops at a Crossroad: HiTech or Else?. ASABE Resour. Magazine 15(6), 5-6.

Cano, E., Horton, R., Liljegren, C., and Bulanon, D.M. 2017. Comparison of Small Unmanned Aerial Vehicles Performance Using Image Processing. J. Imaging, 2017, 3, 4.

DroneDeploy. 2017. Retrieved from https://www.dronedeploy.com/ (Accessed on August 25, 2017)

Event38 Unmanned Systems. 2017. Custom NGB Filter Glass for DIY Camera Conversion, https://event38.com/product/custom-ngb-filter-glass-for-diy-camera-conversion/ (Accessed on June 29, 2017)

Food and Agriculture Organization of the United Nations. 2009. 2050: A third more mouths to feed. http://www.fao.org/news/story/en/item/35571/icode/, (Accessed on March 23, 2017)

Fredembach, C., and Susstrunk, S. 2008. Colouring the near infrared. Proceedings of the IS\&T $16^{\text {th }}$ Color Imaging Conference, pp. 176-182, 2008

Garcia-Ruiz, F., Sankaran, S. Maja, J.M., Lee, W.S., Rasmussen, J., and Ehsani, R. 2013. Comparison of two aerial imaging platforms for identification of Huanglongbing-infected citrus trees, Computers and Electronics in Agriculture 91(2013), 106-115

Kataoka, T., Kaneko, T., Okamoto, H., Terawaki, M., and Hata, S. 2004. Development of Crop Growth Mapping System Using Machine Vision (Part 1) Producing Entire Crop Rows Image by Montaging Continuous Images, Journal of the Japanese Society of Agricultural Machinery. 66(5), pp. 74-82.

Koller, M., and Upadhyaya, S.K. 2005. Relationship between modified normalized 
difference vegetation index and leaf area index for processing tomatoes. Appl. Eng. Agric. 21(5), 927-933.

Lee, W.S., Alchanatis, V., Yang, C., Hirafuji, M., Moshou, D., and Li, C. 2010. Sensing technologies for precision specialty crop production. Computers and Electronics in Agriculture. 74(2010), 2- 33.

Llewellyn Data Processing. 2017. Remote Sensing Cameras, https://www.maxmax.com/maincamerapage/remote-sensing (Accessed on June 29, 2017)

McCabe, M.F., Houborg, R., and Lucieer, A. 2016. High-resolution sensing for precision agriculture: from Earth-observing satellites to unmanned aerial vehicles, Proc. SPIE 9998, Remote Sensing for Agriculture, Ecosystems, and Hydrology XVIII, 999811 (October 25, 2016); DOI:10.1117/12.2241289

MicaSense. 2017. RedEdge, https://www.micasense.com/rededge/ (Accessed on March 23, 2017)

MIDOPT. 2017. DB660/850 Dual Bandpass Red + 850 nm NIR, http://midopt.com/filters/db660850/ (Accessed on March 23, 2017)

Pix4D. 2017. Retrieved from https://pix4d.com/ (Accessed on August 25, 2017)

Sankaran, S., Mishra, A.R., Maja, J.M., and Ehsani, R. 2011. Visible-near infrared spectroscopy for detection of Huanglongbing in citrus orchards. Computers and Electronics in Agriculture, 77(2), 127-134

Yang, C., Everitt, J.H., and Bradford, J.M. 2004. Airborne hyperspectral imagery and yield monitor data for mapping cotton yield variability. Precision Agric. 5(5), 445-461.

Figure Captions

Figure 1 Canon S100 Camera with calibration targets

Figure 2 Micasense RedEdge Multispectral Camera

Figure 3 E384 UAV

Figure 4 Target Apple Orchard

Figure 5 Gray Scale Images of red band and near-infrared band

Figure 6 NDVI Images of both cameras 


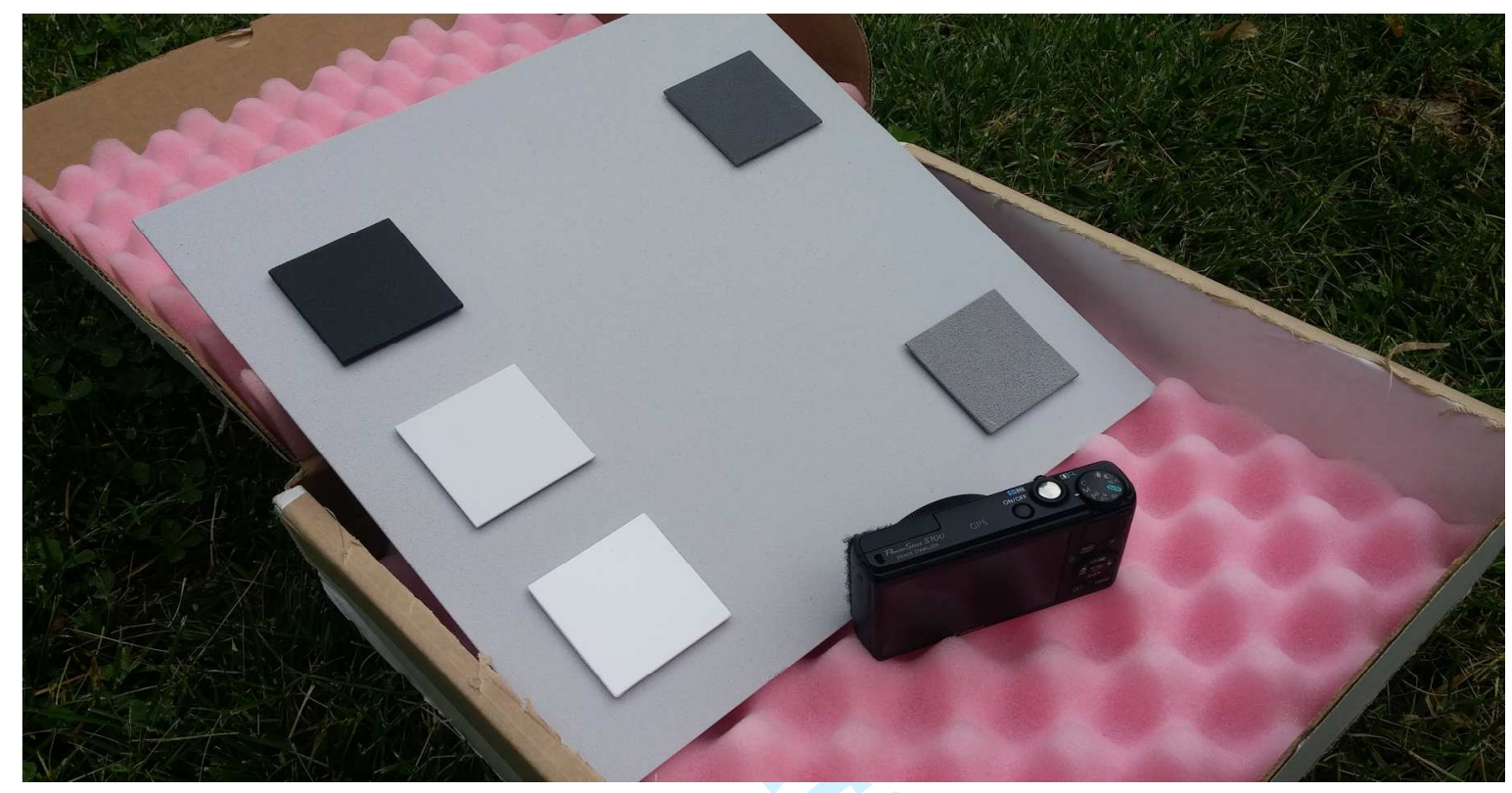

Figure 1 Canon S100 Camera with calibration targets 


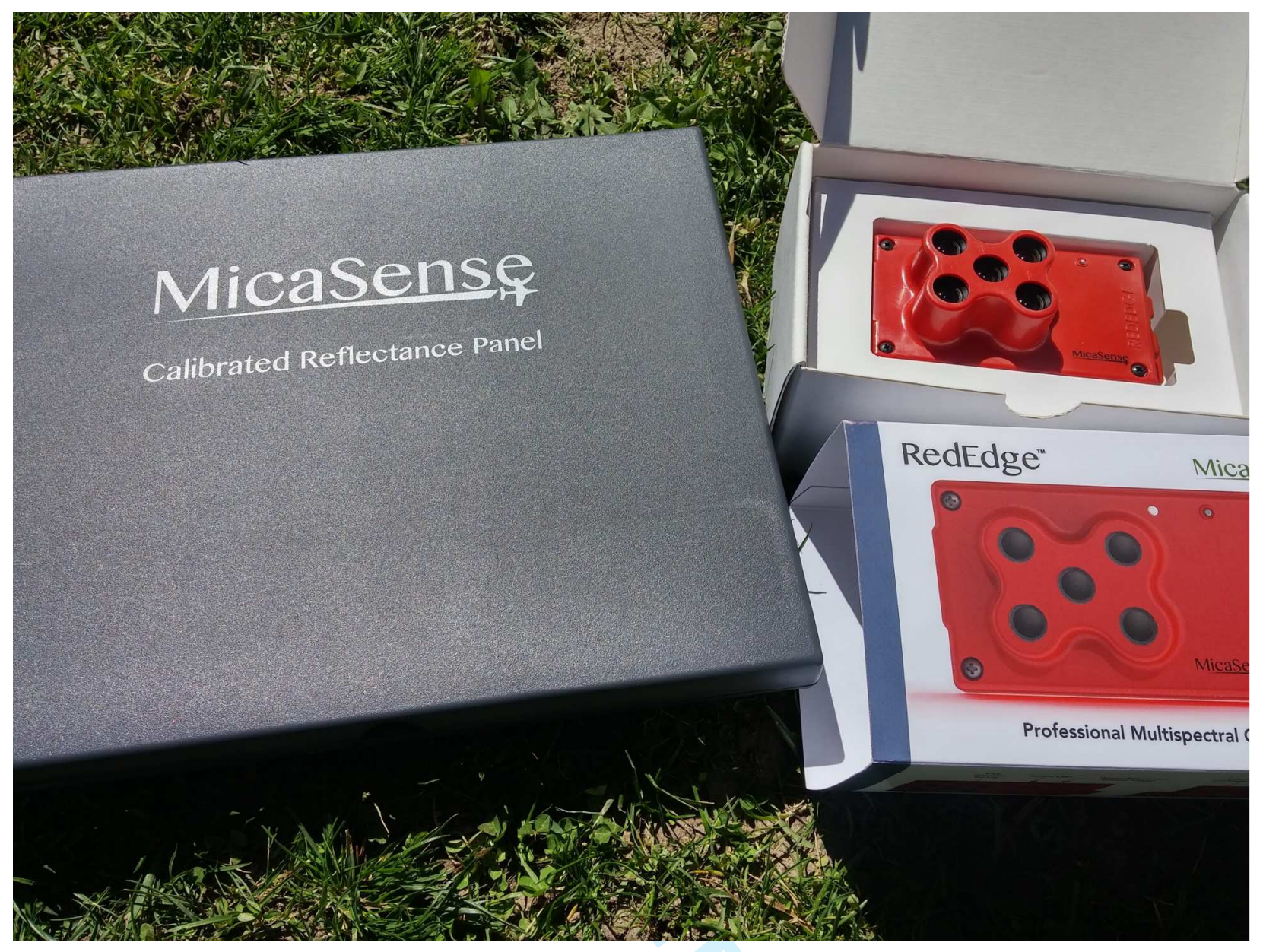

Figure 2 Micasense RedEdge Multispectral Camera 


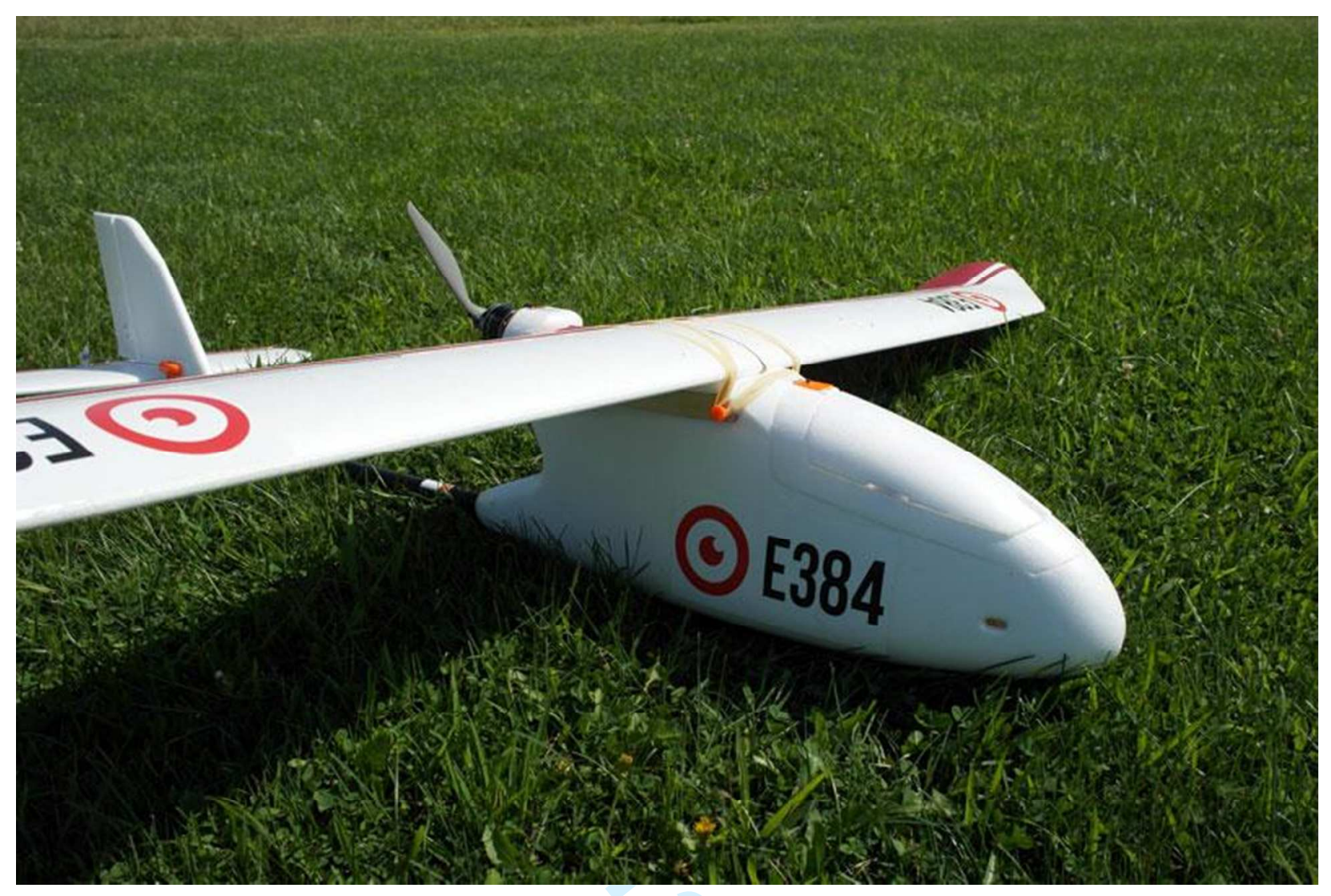

Figure 3 E384 UAV 


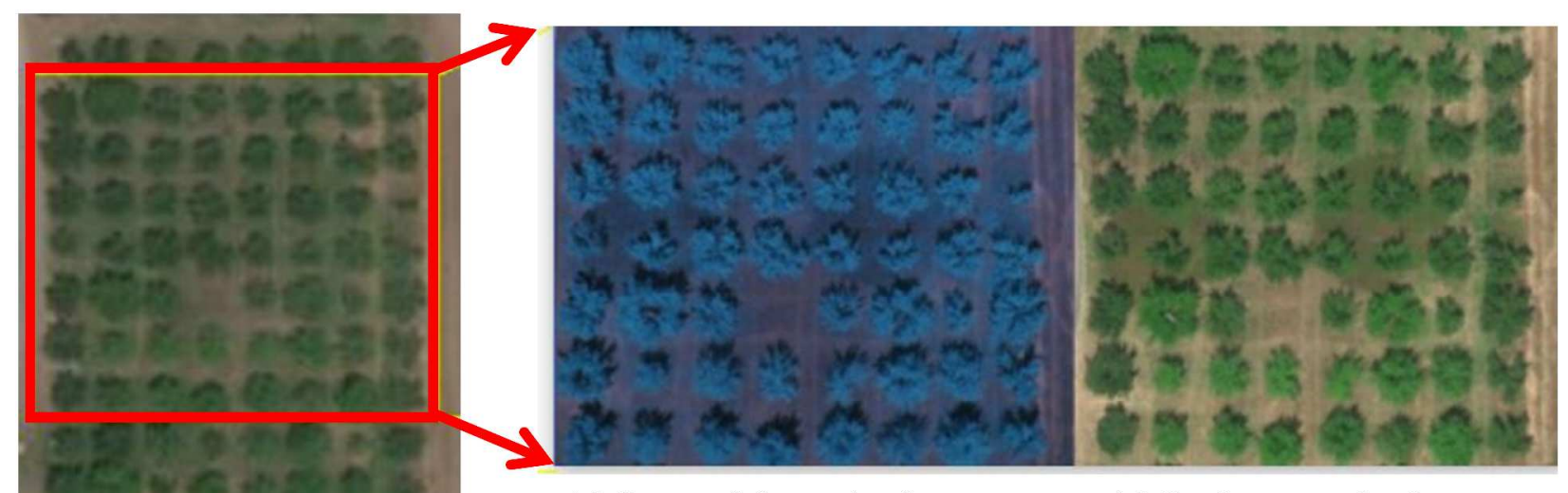

b) Canon false color image c) MicaSense color image

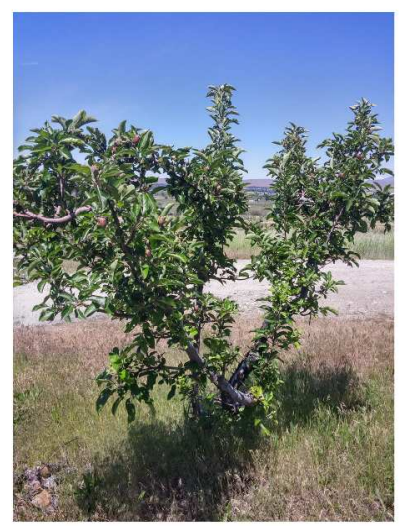

a) Target apple orchard

d) Sample ground image of apple tree

Figure 4 Target Apple Orchard 


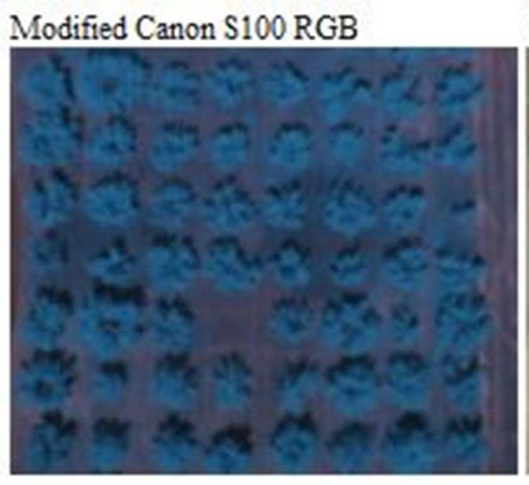

Micasense RedEdge 3 RGB

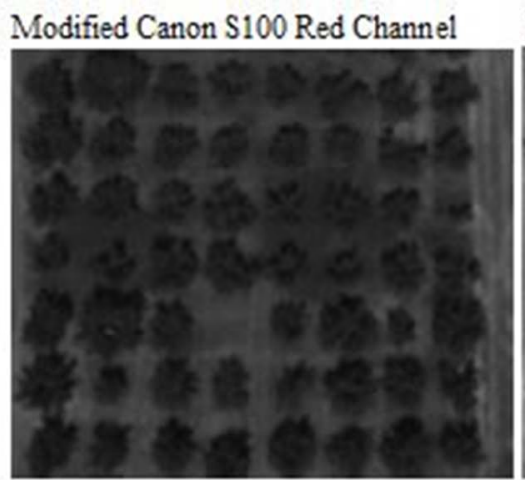

Micasense RedEdge 3 Red Channel
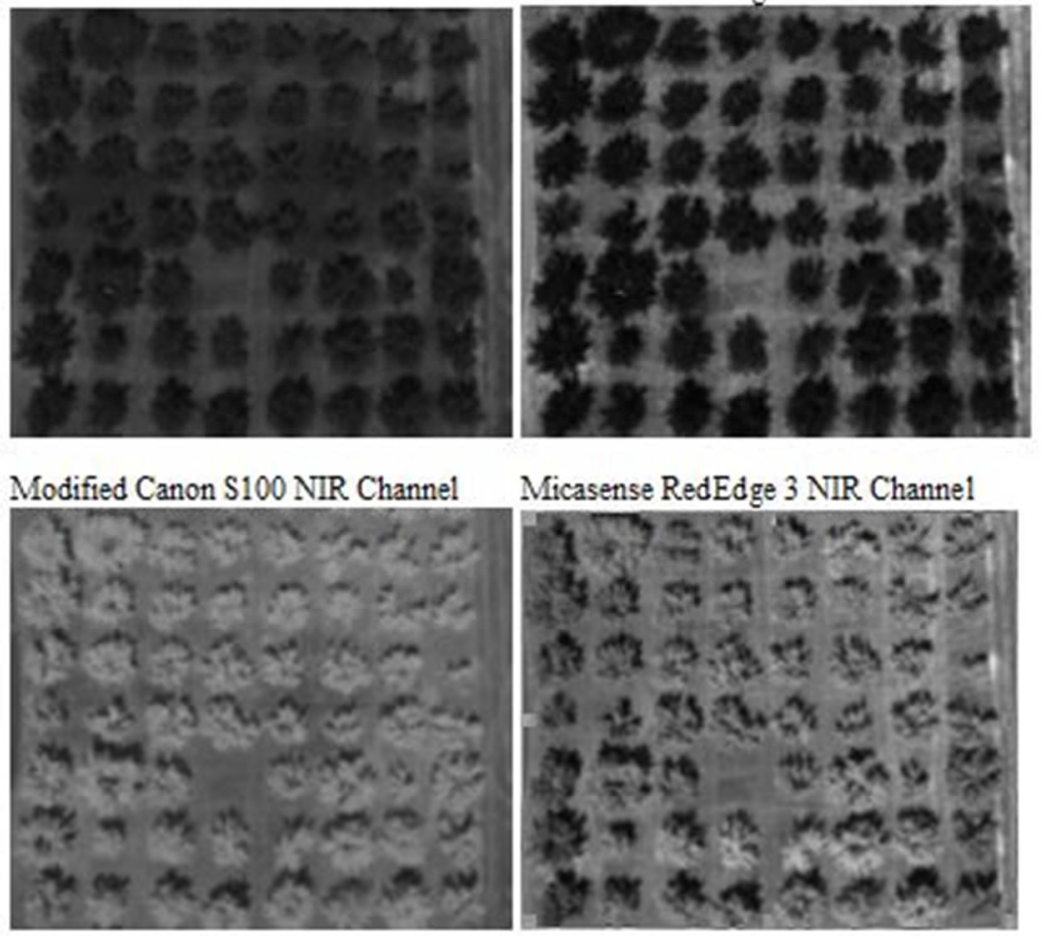

Micasense RedEdge 3 NIR Channel

Figure 5 Gray Scale Images of red band and near-infrared band 


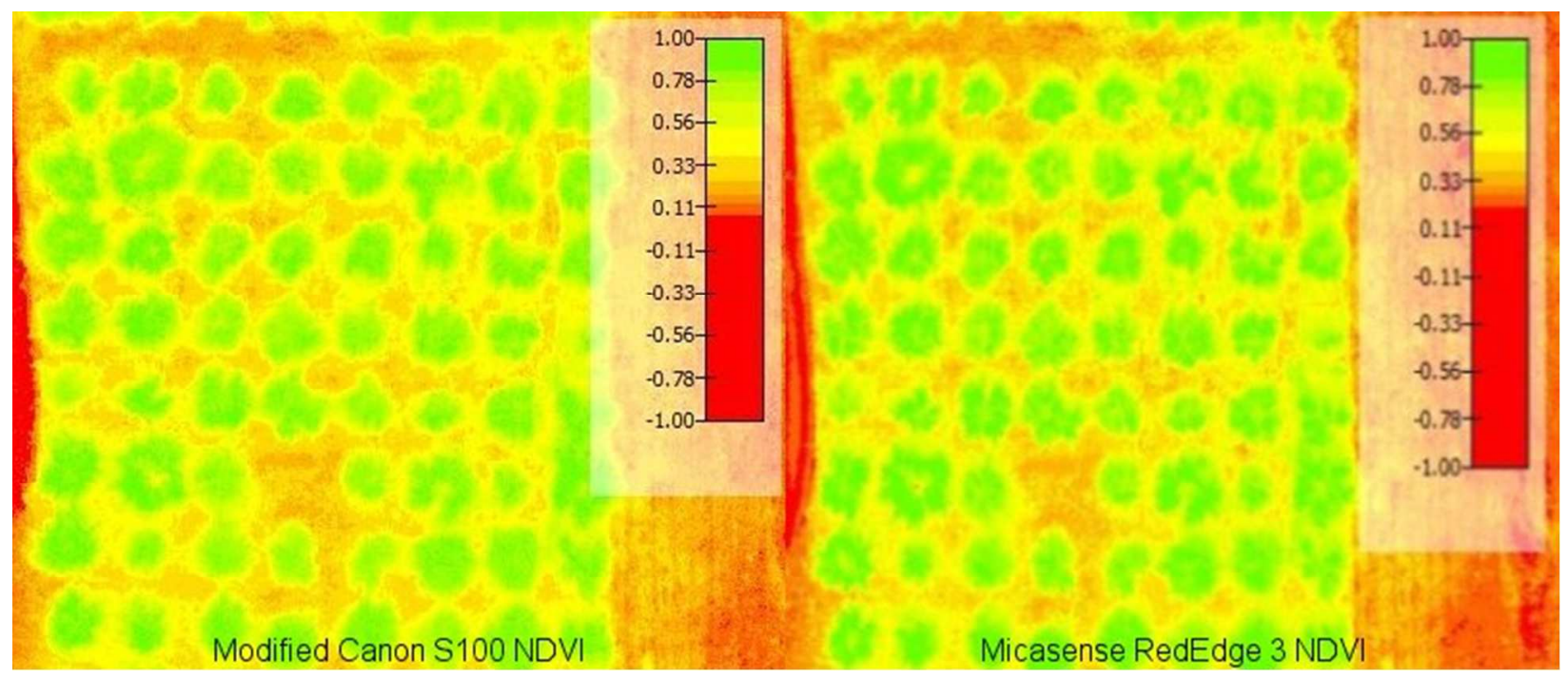

Figure 6 NDVI Images of both cameras 